\title{
Quantum mechanics from a Heisenberg-type equality
}

\author{
Michael J. W. Hall ${ }^{1}$ and Marcel Reginatto ${ }^{2}$ \\ 1 Theoretical Physics, RSPSE \\ Australian National University \\ Canberra ACT 0200 Australia. \\ michael.hall@anu.edu.au \\ 2 Physikalisch-Technische Bundesanstalt \\ Bundesallee 100 \\ 38116 Braunschweig Germany
}

\begin{abstract}
The usual Heisenberg uncertainty relation, $\Delta X \Delta P \geq \hbar / 2$, may be replaced by an exact equality for suitably chosen measures of position and momentum uncertainty, which is valid for all wavefunctions. This exact uncertainty relation, $\delta X \Delta P_{n c} \equiv \hbar / 2$, can be generalised to other pairs of conjugate observables such as photon number and phase, and is sufficiently strong to provide the basis for moving from classical mechanics to quantum mechanics. In particular, the assumption of a nonclassical momentum fluctuation, having a strength which scales inversely with uncertainty in position, leads from the classical equations of motion to the Schrödinger equation.
\end{abstract}

\section{An exact uncertainty relation}

The well known Heisenberg inequality $\Delta X \Delta P \geq \hbar / 2$ has a fundamental significance for the interpretation of quantum theory - as argued by Heisenberg himself, it provides "that measure of freedom from the limitations of classical concepts which is necessary for a consistent description of atomic processes" [1]. It might be asked whether this "measure of freedom" from classical concepts can be formulated more precisely. The answer is, surprisingly, yes - and, as a consequence, the Heisenberg inequality can be replaced by an exact equality, valid for all pure states.

To obtain this equality, note that for a classical system, the position and momentum observables can be measured simultaneously, to an arbitrary accuracy. For a quantum system we therefore define the classical component of the momentum to be that observable which is closest to the momentum observable, under the constraint of being comeasurable with the position of the system. More formally, for the case that we have maximal knowledge about the system, i.e., we know its wavefunction $\psi(x)$, the classical component $P_{c l}^{\psi}$ of the momentum is defined by the properties

$$
\left[X, P_{c l}^{\psi}\right]=0, \quad\left\langle\left(P-P_{c l}^{\psi}\right)^{2}\right\rangle_{\psi}=\text { minimum } .
$$


The unique solution to (11) is $P_{c l}^{\psi}=\int d x|x\rangle\langle x| P_{c l}^{\psi}(x)$, where [2]

$$
P_{c l}^{\psi}(x)=\frac{\hbar}{2 i}\left[\psi^{\prime}(x) / \psi(x)-\psi^{* *}(x) / \psi^{*}(x)\right] .
$$

Thus $P_{c l}^{\psi}(x)$ provides the best possible estimate of momentum for state $\psi(x)$ consistent with position measurement result $X=x$. It may also be recognised as the momentum current which appears in the quantum continuity equation for the probability density $|\psi(x)|^{2}$, as well as the momentum associated with the system in the de Broglie-Bohm interpretation of quantum mechanics [3].

Having a classical momentum component, it is natural to define the corresponding nonclassical component of the momentum, $P_{n c}^{\psi}$, via the decomposition

$$
P=P_{c l}^{\psi}+P_{n c}^{\psi}
$$

The average of $P_{n c}^{\psi}$ is zero for state $\psi(x)$, and hence $P$ may be thought of as comprising a nonclassical fluctuation about a classical average. It is the nonclassical component which is responsible for the commutation relation $[X, P]=i \hbar$. One has the related decomposition [2]

$$
(\Delta P)^{2}=\left(\Delta P_{c l}^{\psi}\right)^{2}+\left(\Delta P_{n c}^{\psi}\right)^{2}
$$

of the momentum variance into classical and nonclassical components, and there is a similar decomposition of the kinetic energy.

The magnitude of the nonclassical momentum fluctuation, $\Delta P_{n c}^{\psi}$, provides a natural measure for that "degree of freedom from the limitations of classical concepts" referred to by Heisenberg [1]. Note that this magnitude can be operationally determined from the statistics of $X$ and $P$, via equations (2) and (4).

It is remarkable that $\Delta P_{n c}^{\psi}$ satisfies an exact uncertainty relation [2]

$$
\delta X \Delta P_{n c}^{\psi} \equiv \hbar / 2
$$

where $\delta X$ denotes a classical measure of position uncertainty, called the "Fisher length", defined via [4]

$$
(\delta X)^{-2}=\int_{-\infty}^{\infty} d x p(x)[(d / d x) \ln p(x)]^{2}
$$

for probability density $p(x)$. There is thus a precise connection between the statistics of complementary observables.

The exact uncertainty relation (5) is far stronger than the usual Heisenberg inequality (the latter follows immediately via (4) and the Cramer-Rao inequality $\Delta X \geq \delta X$ of classical statistical estimation theory). For example, suppose that at some time the wavefunction $\psi(x)$ is confined to some interval. Then, since $\ln p(x)$ changes from $-\infty$ to a finite value in any neighbourhood containing an endpoint of the interval, the Fisher length $\delta X$ vanishes. The exact uncertainty relation (5) thus immediately implies that $\Delta P_{n c}^{\psi}$, and hence $\Delta P$, is unbounded for any such confined wavefunction.

Exact uncertainty relations may be generalised and/or applied to, for example, density operators, higher dimensions, energy bounds, photon number and phase, and entanglement [2]. A conjugate relation, $\delta P \Delta X_{n c}^{\psi} \equiv \hbar / 2$, may also be derived. In the following section the very existence of exact uncertainty relations is used as a basis for deriving the Schrödinger equation. 


\section{QM from an exact uncertainty principle}

Landau and Lifschitz wrote, referring to the Heisenberg uncertainty principle, that "this principle in itself does not suffice as a basis on which to construct a new mechanics of particles" [5]. However, the existence of exact uncertainty relations for quantum systems raises anew the question of whether the uncertainty principle, at the conceptual core of the standard Copenhagen interpretation of quantum theory, can be put in a form strong enough to provide an axiomatic means for moving from classical to quantum equations of motion. The corresponding exact uncertainty principle would thus be on a par with alternative derivations based on the principle of superposition, $C^{*}$-algebras, quantum logics, etc.

It has recently been shown that an "exact uncertainty principle" does indeed exist, where the Schrödinger equation may be derived via the postulate that classical systems are subjected to random momentum fluctuations of a strength inversely proportional to uncertainty in position [6]. This new approach is summarised below (though using a different method of proof than in [6]).

Now, in any axiomatic-type construction of quantum mechanics one must first choose a classical starting point, to be generalised or modified appropriately. The starting point here is a statistical one - the classical motion of an ensemble of particles - and indeed most of the assumptions to be made below will be seen to have a statistical character.

Consider then a classical ensemble of $n$-dimensional particles of mass $m$ moving under a potential $V$. The motion may be described via the Hamilton-Jacobi and continuity equations

$$
\frac{\partial s}{\partial t}+\frac{1}{2 m}|\nabla s|^{2}+V=0, \quad \frac{\partial p}{\partial t}+\nabla \cdot\left[p \frac{\nabla s}{m}\right]=0,
$$

respectively, for the "momentum potential" $s$ and the position probability density $p$. These equations follow from the variational principle $\delta L=0$ with Lagrangian

$$
L=\int d t d^{n} x p\left[\frac{\partial s}{\partial t}+\frac{1}{2 m}|\nabla s|^{2}+V\right],
$$

under fixed endpoint variation with respect to $p$ and $s$. This Lagrangian is therefore chosen as our classical starting point.

It is now assumed that the classical Lagrangian (6) must be modified, due to the existence of random momentum fluctations. The nature of these fluctuations is not important to the argument - they may be postulated to model experimental evidence that the momentum variance is sometimes greater than the expected $\int d x p|\nabla s-\langle\nabla s\rangle|^{2}$; or they may be regarded as a device to make the system irreducibly statistical (since such fluctuations imply the velocity relation $\mathbf{v}=m^{-1} \nabla s$ no longer holds, and hence cannot be integrated to give corresponding trajectories). The assumption is simply that the momentum associated with position $x$ is given by

$$
\mathbf{P}=\nabla s+\mathbf{N}
$$

where the fluctuation term $\mathbf{N}$ vanishes on the average at each point $x$. The physical meaning of $s$ thus changes to being an average momentum potential.

It follows that the average kinetic energy $\left\langle|\nabla s|^{2}\right\rangle /(2 m)$ appearing in (6) should be replaced by $\left\langle|\nabla s+\mathbf{N}|^{2}\right\rangle /(2 m)$ (where \langle\rangle now denotes the average over fluctuations and position), giving rise to the modified Lagrangian

$$
L^{\prime}=L+(2 m)^{-1} \int d t\langle\mathbf{N} \cdot \mathbf{N}\rangle=L+(2 m)^{-1} \int d t(\Delta N)^{2},
$$


where $\Delta N=\langle\mathbf{N} \cdot \mathbf{N}\rangle^{1 / 2}$ is a measure of the strength of the fluctuations. Note that the additional term in (7) corresponds to the kinetic energy of the fluctuations, and so is positive.

The additional term is specified uniquely, up to a multiplicative constant, by the following three assumptions:

(1) Action principle: $L^{\prime}$ is a scalar Lagrangian with respect to the fields $p$ and $s$, where the variational principle $\delta L^{\prime}=0$ yields causal equations of motion. Thus

$$
(\Delta N)^{2}=\int d^{n} x p f(p, \nabla p, \partial p / \partial t, s, \nabla s, \partial s / \partial t, \mathbf{x}, t)
$$

for some scalar function $f$.

(2) Additivity: If the system comprises two independent non-interacting subsystems 1 and 2 , with $p=p_{1} p_{2}$, then the Lagrangian decomposes into additive subsystem contributions. Thus

$$
f=f_{1}+f_{2} \quad \text { for } \quad p=p_{1} p_{2},
$$

where subscripts denote corresponding subsystem quantities. Note this is equivalent to the statistical assumption that the corresponding subsystem fluctuations are linearly uncorrelated, i.e., $\left\langle\mathbf{N}_{\mathbf{1}} \cdot \mathbf{N}_{\mathbf{2}}\right\rangle=0$.

(3) Exact uncertainty principle: The strength of the momentum fluctuation at any given time is determined by, and scales inversely with, the uncertainty in position at that time. Thus

$$
\Delta N \rightarrow k \Delta N \quad \text { for } \quad \mathbf{x} \rightarrow \mathbf{x} / k,
$$

and moreover, since position uncertainty is entirely characterised by the probability density $p$ at a given time, the function $f$ cannot depend on $s$, nor explicitly on $t$, nor on the time-derivative of $p$.

We now have the following theorem [6]:

Theorem: The above three assumptions of an action principle, additivity, and an exact uncertainty principle imply that

$$
(\Delta N)^{2}=C \int d^{n} x p|\nabla \ln p|^{2},
$$

where $C$ is a positive universal constant.

A (new) proof of the above theorem is outlined below. Here its main consequence is noted. In particular, it follows directly via (7) that the equations of motion for $p$ and $s$, corresponding to the variational principle $\delta L^{\prime}=0$, can be expressed as the single complex equation

$$
i \hbar \frac{\partial \psi}{\partial t}=-\frac{\hbar^{2}}{2 m} \nabla^{2} \psi+V \psi
$$

where one defines $\hbar:=2 \sqrt{C}$ and $\psi:=\sqrt{p} e^{i s / \hbar}$. Thus the above postulates yield equations of motion equivalent to the Schrödinger equation. It can be shown that the mapping from fields $p$ and $s$ to the wavefunction $\psi$ arises naturally from seeking canonical transformations which map to "normal modes" of the system [6]. It is remarkable that a linear equation results from the assumptions used.

\section{Proof of theorem}

To see how the above theorem follows, note first that the action principle and exact uncertainty principle imply that the scalar function $f$ depends only on $p, \nabla p$, and $\mathbf{x}$. It 
can therefore be written in the form

$$
f=g\left(u, v, w, r^{2}\right)
$$

where

$$
u=\ln p, \quad v=(\mathbf{x} \cdot \nabla p) / p, \quad w=|\nabla p|^{2} / p^{2}, \quad r^{2}=\mathbf{x} \cdot \mathbf{x} .
$$

For $p=p_{1} p_{2}$ one finds $u=u_{1}+u_{2}, v=v_{1}+v_{2}$, and $w=w_{1}+w_{2}$, and hence the additivity assumption implies that

$$
g\left(u_{1}+u_{2}, v_{1}+v_{2}, w_{1}+w_{2}, r_{1}^{2}+r_{2}^{2}\right)=g_{1}\left(u_{1}, v_{1}, w_{1}, r_{1}^{2}\right)+g_{2}\left(u_{2}, v_{2}, w_{2}, r_{2}^{2}\right) .
$$

Thus $g$ must be linear in $u, v, w$ and $r^{2}$, yielding

$$
f=A \ln p+B \frac{\mathbf{x} \cdot \nabla p}{p}+C \frac{|\nabla p|^{2}}{p^{2}}+D \mathbf{x} \cdot \mathbf{x},
$$

where $A, B, C$ and $D$ are universal constants. Note that the first term corresponds to an entropic potential in the Lagrangian $L^{\prime}$.

Finally, noting that $p(x) \rightarrow k^{n} p(k x)$ under the transformation $\mathbf{x} \rightarrow \mathbf{x} / k$, the exact uncertainty principle forces $A=B=D=0$, and the theorem is proved (where the positivity of $C$ follows from the positivity of $\left.(\Delta N)^{2}\right)$.

\section{Conclusions}

It has been shown that the Heisenberg uncertainty relation may be upgraded to an exact uncertainty relation, and that a corresponding exact uncertainty principle may be used as the single nonclassical element necessary for obtaining the Schrödinger equation. Thus the uncertainty principle is able to provide not only a conceptual underpinning of quantum mechanics, but an axiomatic underpinning as well. The above approach immediately generalises to include electromagnetic potentials, and work on generalisations to systems with spin and to quantum fields is in progress.

The form chosen for the exact uncertainty principle, that classical systems are subject to random momentum fluctuations of a strength inversely proportional to uncertainty in position, is of course motivated by the momentum decomposition (3) and exact uncertainty relation (5) holding for quantum systems. Thus, not surprisingly, there are a number of connections between the latter uncertainty relation and the Theorem of section 2. For example, for a 1-dimensional system, the Theorem immediately implies the uncertainty relation

$$
\delta X \Delta N=\sqrt{C}=\hbar / 2
$$

for the momentum fluctations, which may be compared to (5).

The exact uncertainty principle has a type of "nonlocality" built into it: the form of $\Delta N$ specified by the Theorem implies that a change in the position probability density arising from actions on one subsystem (eg, a position measurement), will typically influence the behaviour of a second subsystem correlated with the first. This nonlocality corresponds to quantum entanglement, and has been analysed to some extent via exact uncertainty relations in [2].

It is worth noting that the approach here, based on exact uncertainty, is rather different from other approaches which assign physical meaning to fields $p$ and $s$ related to the

wavefunction. For example, in the de Broglie-Bohm approach [3], there are no momentum 
fluctuations, and the classical equations of motion for $p$ and $s$ are instead modified by adding a mass-dependent "quantum potential", $Q$, to the classical potential term in the Hamilton-Jacobi equation. The form of this quantum potential is left unexplained, and is interpreted as arising from the influence of an associated wave acting on the system. In contrast, in the exact uncertainty approach $\nabla s$ is an average momentum, the form of an additional kinetic energy term arising from random momentum fluctuations is derived, and no associated wave is assumed. The formal connection between the two approaches is the relation

$$
\delta\left(L^{\prime}-L\right)=\int d t d^{n} x Q \delta p .
$$

Finally, the exact uncertainty approach is also very different from the stochastic mechanics approach [7]. The latter postulates the existence of a classical stochastic process in configuration space, with a drift velocity assumed to be the gradient of some scalar, and defines an associated time-symmetric "mean acceleration" $\mathbf{a}$ in terms of averages over both the stochastic process and a corresponding time-reversed process, which is postulated to obey Newton's law $m \mathbf{a}=-\nabla V$. In contrast, the exact uncertainty approach does not rely on a classical model of fluctuations, nor on a new definition of acceleration, nor on properties of stochastic processes running backwards in time. The formal connections between the approaches are

$$
\nabla s=m \mathbf{u}, \quad(\Delta N)^{2}=m^{2}\langle\mathbf{v} \cdot \mathbf{v}\rangle,
$$

where $\mathbf{u}+\mathbf{v}$ and $\mathbf{u}-\mathbf{v}$ are the drift velocities of the forward-in-time and backward-in-time processes respectively. It should be noted that $\langle\mathbf{u} \cdot \mathbf{v}\rangle \neq 0$, and hence one cannot identify $m \mathbf{v}$ with the random momentum fluctuation $\mathbf{N}$.

\section{References}

[1] W. Heisenberg, The Physical Principles of the Quantum Theory (Dover, USA, 1930), page 4 .

[2] M.J.W. Hall, Phys. Rev. A 64 (2001) 052103 quant-ph/0107149.

[3] D. Bohm, Phys. Rev. 85 (1952) 143, 187.

[4] R.A. Fisher, Proc. Cambridge Philos. Soc. 22 (1925) 700.

[5] L.D. Landau and E.M. Lifschitz, Quantum Mechanics, 3rd edition (Pergamon, Oxford, 1977), page 2 .

[6] M.J.W. Hall and M. Reginatto quant-ph/0102069.

[7] E. Nelson, Phys. Rev. 150 (1966) 1079; E. Nelson, Dynamical Theories of Brownian Motion (Princeton University Press, USA, 1967). 